\title{
Correction to: Garnet major-element composition as an indicator of host-rock type: a machine learning approach using the random forest classifier
}

\author{
Jan Schönig ${ }^{1}$ (I) $\cdot$ Hilmar von Eynatten ${ }^{1} \cdot$ Raimon Tolosana-Delgado $^{2} \cdot$ Guido Meinhold $^{1,3,4}$
}

Published online: 14 December 2021

○) Springer-Verlag GmbH Germany, part of Springer Nature 2021

\section{Correction to: \\ Contributions to Mineralogy and Petrology (2021) 176:98 \\ https://doi.org/10.1007/s00410-021-01854-w}

On account of a programming bug, the replacement of logratios with a value below detection limit in the nominator and/or denominator as described in the "Model development" section of the original article has been incorrectly applied in the provided 'garnetRF v1.0' web application. This has been patched and since 22nd November 2021 the revised web application 'garnetRF v1.1' is available via the link given in the original article (http://134.76.17.86:443/ garnetRF/). The correction does not influence the presented discrimination model in any respect. However, the predictions made by the web application are affected. Because the 'garnetRF v1.0' web application has solely been used for predictions in the "Sensitivity and applicability" section of the original article, the section and the corresponding Fig. 9 have been revised according to the predictions given by the revised web application 'garnetRF v1.1'. Observations and statements made in the original article are only affected in case of the example study from the Almklovdalen area. Revised predictions in this case do not assign detrital garnet to a mantle host rock, and accordingly this example study has been removed from the main text and Fig. $9 \mathrm{~b}$. All predictions according to the other examples have been corrected in Fig. 9 and the main text has been adjusted, which has no influence on the statements made. Section "Sensitivity and applicability" and the corresponding Fig. 9 have been updated in the original article by the revised versions.

Publisher's Note Springer Nature remains neutral with regard to jurisdictional claims in published maps and institutional affiliations.

The original article can be found online at https://doi.org/10.1007/ s00410-021-01854-w.

Jan Schönig

jan.schoenig@uni-goettingen.de

1 Geoscience Centre Göttingen, University of Göttingen, Goldschmidtstraße 3, 37077 Göttingen, Germany

2 Helmholtz-Zentrum Dresden-Rossendorf, Helmholtz Institute Freiberg for Resources Technology, Chemnitzer Straße 40, 09599 Freiberg, Germany

3 School of Geography, Geology and the Environment, Keele University, Keele, Staffordshire ST5 5BG, UK

4 Institute for Geology, TU Bergakademie Freiberg, Bernhard-von-Cotta-Straße 2, 09599 Freiberg, Germany 